\title{
Acoustic Radiation Force Optical Coherence Elastography
}

\author{
Zhongping Chen \\ Beckman Laser Institute, Department of Biomedical Engineering \\ University of California Irvine, Irvine, CA 92617
}

We report on the development of an acoustic radiation force optical coherence elastography (ARF-OCE) technology to characterize tissues biomechanical properties. Knowledge of tissue mechanical properties provides valuable medical information in disease diagnosis and prognosis. There is a close correlation between tissue elasticity and pathology. For example, in atherosclerosis, measurement of tissue biomechanical properties has the potential to differentiate between various plaque components. Furthermore, tissue mechanical properties provide critical information to assess the vulnerability of plaques. The stress in the cap increases with decreasing thickness and increasing macrophage infiltration. High strain locations in the vessel wall indicate the presence of vulnerable plaques. We have applied the ARF-OCE to image postmortem human coronary artery with atherosclerosis. The result demonstrates the potential of ARF-OCE as a non-invasive method for imaging and characterizing vulnerable plaques. In addition, we have developed an acoustic radiation force orthogonal excitation optical coherence elastography (ARFOE-OCE) method for the visualization of the shear wave and the calculation of the shear modulus based on the Doppler OCT variance method. The ARF-OCE technology will have a broad range of clinical applications, including imaging and characterizing cardiovascular atherosclerotic lesions, imaging and diagnosing of early stage cancer, and imaging and evaluating ophthalmic diseases such as keratoconus and age-related macular degeneration. 